\title{
Procedures Supplemental Qualifiers Dataset
}

National Cancer Institute

\section{Source}

National Cancer Institute. Procedures Supplemental Qualifiers Dataset. NCI Thesaurus.

Code C147239.

A dataset containing supplemental information, specifically non-standard variables, to parent records in the procedures domain. 